This item was submitted to Loughborough's Research Repository by the author.

Items in Figshare are protected by copyright, with all rights reserved, unless otherwise indicated.

\title{
Design re-use: critical application of healthcare building design evidence
}

PLEASE CITE THE PUBLISHED VERSION

https://doi.org/10.1108/ECAM-07-2017-0118

PUBLISHER

(C) Emerald

VERSION

AM (Accepted Manuscript)

PUBLISHER STATEMENT

This work is made available according to the conditions of the Creative Commons Attribution-NonCommercial 4.0 International (CC BY-NC 4.0) licence. Full details of this licence are available at: http://creativecommons.org/licenses/by-nc/4.0/

\section{LICENCE}

CC BY-NC 4.0

\section{REPOSITORY RECORD}

Wanigarathna, Nadeeshani, F. Sherratt, Andrew Price, and Simon Austin. 2019. "Design Re-use: Critical Application of Healthcare Building Design Evidence". figshare. https://hdl.handle.net/2134/34838. 


\title{
Design re-use: Critical application of healthcare building design evidence
}

\author{
Nadeeshani Wanigarathna
}

Department of Engineering and the Built Environment, Anglia Ruskin University, City, UK

Bishop Hall Lane, Chelmsford CM1 1SQ.

Nadeeshani.wanigarathna@anglia.ac.uk

Telephone: 00447413333269

\section{Fred Sherratt}

Department of Engineering and the Built Environment, Anglia Ruskin University, City, UK

Bishop Hall Lane, Chelmsford CM1 1SQ.

Andrew Price

School of Civil and Building Engineering, Loughborough University, City, UK

Epinal Way, Loughborough LE11 3TU.

Simon Austin

School of Civil and Building Engineering, Loughborough University, City, UK

Epinal Way, Loughborough LE11 3TU.

\section{Acknowledgement}

The work was supported by ESPRC IMCRC under grant [EP/E002323/1] and under grant EPSRC HaCIRIC [EP/D039614/1 and EP/I029788/1]. The authors also wish to acknowledge the industry partners who participated in the case studies. 


\section{Abstract}

Purpose: Re-use of good design solutions is a key source of evidence and knowledge in the design of healthcare buildings. However, due to the unique nature of healthcare built environments, critical application of this evidence is of paramount important. This research investigated the features of such critical application, and identified the aspects that need to be considered during the re-use of good designs.

Design/methodology/approach: Data from three case studies of hospital designs in the UK were used to explore the processes behind the adaption and re-use of design solutions during the design of healthcare buildings. Data were thematically analysed to distinguish the aspects that should be carefully compared and contrasted during design re-use.

Findings: Existing designs of healthcare buildings should be captured and evaluated along with: patient demographics, care models of the hospital, other local departmental needs, and facility operational aspects in order to ensure the effectiveness of re-use. In addition, properly introducing the design to the users is also a part of successful design re-use.

Practical implications: This research provides details of how healthcare built environment designs are embedded in project-unique circumstances. The results could therefore be used to develop meaningful and informative evaluation mechanisms for new and re-used healthcare building design features.

Originality/value: This research extends the understanding of critical application of healthcare design evidence, by explaining how healthcare design solutions should be evaluated during the design process. 
Limitations: The findings of this research was integrated into a framework to support healthcare designers on effective re-use of good designs. This data driven framework could be validated further with design practitioners. Further, this research relied on memory recall of the interviewees and the accuracy and completeness of documentary records.

Keywords: healthcare built environments; design re-use; critical application of evidence, design evaluation

\section{Introduction}

They say that 'all design is redesign'. An average designer, whether consciously or subconsciously, draws from an ensemble of internal and external previously designed artefacts or components (Frutcher and Demian, 2002, Gunduz and Yetisir 2016). Designers retrieve existing solutions that historically solved similar problems examine such existing design solutions and reflect on how they could be applied to the current design problem under scrutiny (Sönmez 2018) through various means, to ultimately adopt, adapt with major or minor revisions or even reject the proposed solutions ( Mahar and Pu, 1997; Yu et al., 2012). Design scholars refer this process as 'design re-use'. Researchers have claimed that design reuse is more cost effective than recreating similar designs for new design problems; as it facilitates the dissemination of innovative solutions and sharing best practices (Wanigarathna, 2014) and is an effective approach to increasing design productivity levels (Gunduz and Yetisir 2016). Furthermore, the engineering construction sector has a culture of re-using standards components throughout the supply chain, precisely to increase efficiency and productivity (Fotwe, et al., 2004).

The recent movement in healthcare design, that of Evidence-based Design (EBD), promotes the re-use of good designs or design features. In particular, EBD is an approach to built-environment designing which emphasises the importance of the critical application of 
robust, evidence-based therapeutic features into designs (Hamilton and Watkins, 2009; Moore and Geboy, 2010). The evidence application process is itself an important element of EBD. However, existing research into EBD is not fully able to explain how to critically apply good design features to design problems. Existing literature on the critical application of EBD has put a greater emphasis on how to evaluate evidence to identify good therapeutic design features (such as credibility and completeness of evidence), whilst analysis of their subsequent application is often limited to a discussion of the availability of resources and skills of design practices and designers.

Evidence for EBD comes from both internal and external design knowledge repositories. EBD particularly promotes sharing evidence of good design features (Hamilton, 2003) and using credible design features published externally, therefore the evidence base (or a store of good design solutions) naturally entails a significant levels of external design reuse. However, Fruchter and Demian (2002) claim that external knowledge re-use often fails due to the lack of access to contextual or informal knowledge, such as the rationale behind design decisions, or the interaction between team members on a design team during the design re-use process. Furthermore, healthcare designers re-use EBD design features from previously completed designs contained in the organisational repositories. When reviewing the effectiveness of in-house design solution repositories, Regli, et al. (2000) claimed that previously used design solutions stored in these repositories also often lack supplementary information associated with design rationale, and thus cannot guarantee effective re-use.

Therefore, central to the effectiveness of the practice of design re-use is the level of supplementary contextual details to be considered along with good design features in order to facilitate critical and beneficial application in any later re-use. The aim of this paper is to unpick the details of the design process and designers' rationale when re-using design solutions, with due consideration of both criticality and contextuality. The research is 
supported by the data from 3 in-depth case studies of healthcare building design. The results provide useful insights into the supplementary details that should be captured along with good design features in order to facilitate effective re-use by subsequent users.

\section{Literature review}

\section{Evidence-based design and design re-use}

Evidence of therapeutic design features can be accessed through published sources such as journals or research evidence databases, visiting existing hospitals with therapeutic features, and networking with professionals engage in healthcare design or research. However EBD has not been without criticism, and designers have suggested that EBD will limit creativity and 'bring cookbook architecture to produce dull, repetitious buildings stamped from a mould' (Hamilton, 2003). This argument can be challenged on two grounds: Firstly, the main weakness to this claim is that designers tend to underestimate the extent which they already use previous designs. A recent survey by Taha et al. (2004) found that $100 \%$ of the architects who participated in a survey (from Egypt, Germany, and USA) have reviewed and re-used previous design cases in one way or another. Indeed, 59.5\% of them stated that they always, or most of the time, reviewed previous designs made either by themselves or by others during their design process. They also found that designers spend a significant amount of time searching for past cases, in particular, 216 hours were spent in searching for cases, while 345 hours were spent is studying and analysing them $(38.5 \%$ and $61.5 \%$, respectively). Secondly, built environment design is by and large unique and design solutions cannot simply be copy and pasted from one scenario to another (Kamara et al., 2003). This is particularly relevant for the healthcare sector, since in the UK every National Health Service (NHS) organisation has a unique combination of patient needs, priorities, requirements and resources, making the provision of built environments a complex process in 
which just one single approach does not work (NHS Estates \& Facilities Policy Division, 2013). Many scholars of EBD oppose the 'generic critique' offered by practitioners and instead emphasise that EBD is not an instrumental application of scientifically developed knowledge and theory to confront problems (Moore and Geboy, 2010), but the critical application of best evidence (Hamilton, 2003; Hamilton and Watkins, 2009; Stichler, 2007; Newhouse et al 2007 cited in Stichler 2010) to incorporate therapeutic design features to improve patient and staff outcomes. However, as pointed out in the introduction, existing research into EBD is limited in relation to critical application of during the re-use of therapeutic design features.

\section{Re-use of active and passive design knowlege}

Designers use a variety of approaches, using different knowledge and information sources, to retrieve existing design solutions to develop new design solutions for unique design problems. These come in the forms of both active and passive knowledge. Active knowledge is the knowledge gathered by designers through actually designing themselves or by vicariously experiencing any other existing designs to learn the knowledge embedded in those designs (Wanigarathna, 2014). An example of this would be a designer using their previous memory and knowledge of designing social spaces for hospitals, to subsequently develop a play area for a new hospital project; termed internal memory by Fruchter and Demian (2002). Passive knowledge is the knowledge acquired by designers through secondary sources without directly engaging themselves during designing or vicariously experiencing them (Wanigarathna, 2014). An example would be exemplar solutions presented within published design guidance, or knowledge of innovative solutions gathered during a conference or through any other means of professional networking. It could even be a floor slab designs stored in the designers own organisational design repositories that had been developed by previous designers, for example. Thus, passive knowledge can be further 
categorised into passive knowledge internal to the design organisation and passive knowledge external to the design organisation. Designers use a mix of both active and passive knowledge, gathered through various sources such as other members involved in the design development, supplier and vender information, formal design guidance, published research, visits to existing building (Emmitt, 2007, Wanigarathna, 2014).

However, the effectiveness of design re-use (particularly in the form of passive knowledge) has always been challenged. Fruchter and Demian (2002) claim that passive knowledge re-use in designing often fails compared to active knowledge re-use, due to the lack of access to contextual or informal knowledge, such as the rationale behind design decisions of previous design solutions. Furthermore, Regli, et al. (2000) challenged the effectiveness of in-house organisational design repositories, stating that although most organisations have libraries of previous designs, this alone does not guarantee effective reuse. This is because designs contained in these libraries often contain minimum information such as drawings and specifications associated with the designs, but not other important information associated with design rationale which is required during re-use.

\section{Designers' rationales and knowledge repositories}

It is challenging to decide what information related to the designs should be recorded and stored in such libraries in order to facilitates effective subsequent re-use (Mahar, and De Silva Garza,cited in Hua 2014). Researchers therefore have explored the cognitive aspects of designing in order to identify types of design information requires during effective re-use of design. During reasoning from active knowledge; designers use 'precedents', a form of knowledge embedded in individuals' memories as an episodic memory through direct and vicarious experience of existing designs (Lawson, 2004). Precedent is not about extracting disembodied lessons learned from previous situations and storing these as rules for future designing, instead they are stored as cases and designers reason from cases (Boling, 2010). 
Expert designers accumulate and store a vast numbers of precedents that might come in useful for new design scenarios (Lawson, 2004). They then browse freely and associatively between multiple precedents in order to make relevant connections to create new design solutions (Oxman, 1994). Specific design information gathered by designers during this process goes beyond the drawings and specifications contained in traditional organisational libraries. For example, Kuffner and Ullman (1990, cited in Frucher and Demian, 2002) found that in addition to drawings and specifications, mechanical engineers usually gather information concerning the operation or purpose of a designed object during the re-use of previous designs. Based on the findings from an ethnographic study Demain and Frutcher (2002) found that designers actively consider the context and evolutional history of the design developmental process of previous designs, when they subsequently re-use such design solutions. In a more recent study, Grover, Emmitt and Copping (2017) suggested that designers extract a variety of design information embedded throughout the design development in the form of metaphorical (the cultural, symbolic and experiential context of the project), systemic (spatial, structural and organisational strategies) and elemental (emulation and adaption of parts and conditions embedded in precedent) knowledge of previous designs during their re-use.

A series of research under the term of case-based reasoning (CBR) has also contributed to knowledge in this area. CBR is widely identified as a problem-solving technique that makes analogies between a problem and previously encountered situations (cases) relevant to solving the problem (Kolodner, 1993; Maher and Garza, 1996). Based on these researches, Case-based design systems (CBDSs) are developed to retrieve appropriate cases from a case memory of previous designs to suit a set of requirements/specifications of the new problem (Maher and Balachandran, 1994) and adapt a selected solution(s) to the new design problem. These systems are supported by digital capabilities/artificial intelligence and 
are capable of automating the design process partly or to provide complete automated solutions (ibid). The earliest developed CBDSs were ARCHIE, ARCHIE-II (Domeshek \& Kolodner, 1991). Subsequently, more CBDSs were developed to eliminate weaknesses associated with early systems. More recently, Frucher and Demian and their colleagues (Frucher and Demian 2002) conducted an ethnographic study to explore more details in relation to context and evolution history of designs used by designers during design re-used and incorporated findings to develop CoMem, a CBDS capable of capture knowledge real time with these details.

Other, similar systems developed to be used in the designing of the built environment include:

- MEMORABILIA (Oxman, and Oxman 1993)

- CASECAD, CADSYN (Maher \& Balachandran, 1994)

- GENCAD (Main et al., 2000)

- CADRE, and afterwards IDIOM Swiss Federal Institute of Technology (cited in Taha et al., 2004)

- $\quad$ SEED (Flemming, 1994)

- FABEL cited in Taha et al (supported by the German Ministry for Research and Technology (BMFT) and is carried out by six different organizations and universities in Germany.)

- IDEAs - (IDEAs, 2010)

Most of these repositories were originally intend to empower organisations to create and manage their knowledge assets (Relig et al., 2000) or facilitate design re-use effectively internal within organisations. The aim is to share and reuse design knowledge by capturing and storing design intent, rationale and history in a computer based system (Baxter, et al., 2008). These systems are capable of retaining the cases as ready-made integrated solutions, 
especially for repetitive building types, by making the design process more effective by facilitating the retrieval of precedents and make knowledge available for inexperienced designers and students (Sönmez 2018). Some of these systems (such as Archie III) are capable of facilitating double loop learning (Henderson et al., 2013; Love et al., 2016) by incorporating lessons learnt during the post occupancy evaluation surveys which normally would not reach designers of the project (Gross and Do, 1994).

\section{Developments in the Design Process and design re-use}

Research into design re-use and CBD remains a growing area of research and practice (Baxter, 2008). Some of the most recent advancements include the incorporation of Building Information Modelling (BIM) with design repositories and CBDSs; enabling the live capture of design rationale and information into CBDSs and adoption of CBD into different areas of designs such as landscape design and infrastructure design. Although in its early stages, 2dimensional design information with supplementary descriptions has also contributed to design knowledge reuse and CBDSs (Wang et al., 2002; Hua 2014). The evolution of digital design as a unique field of design knowledge, supported by new technologies provided new insights into the design re-use process (Oxman (2010). Computer Vision and Building Information Modelling can provide large, semantically labelled design repositories for the built environment (Sönmez 2018). For example, Hua (2014) developed a commercial CBR software: TRAMMA program, which is capable of storing and retrieving the 3D models of buildings to facilitate CBR use along with BIM during building design. Demian et al (2016) investigated specific topological relationships which exist in BIM models and how they can be exploited usefully for information retrieval. Furthermore, Sönmez (2018) suggested that novel AI techniques and Similarity-Based Evaluation may contribute to advancements of CBD. In order to eliminate the difficulties associated with time and efforts required to feed information into design repositories, some scholars have developed techniques to live capture 
design information and rationale and store them within design repositories. For example, Chen et al (2007) developed a methodology for live capture and re-use of project knowledge in Construction. As the design of built-environments is a team based activity, part of the design rationale inevitably resides within the social interactions of the team (Senaratne et al., 2017). Live capture methodologies are potentially able to extract these team-generated approaches to design knowledge. However, these methods needs to be supplemented with other strategies to capture lessons learnt during the in-use phase of the buildings, since some of the vital lessons about effective design are only learned from post project reviews (Kululanga and Kuotcha, 2005). Design re-use research has also been conducted in the other areas such as landscape and infrastructure design. Senbel et al. (2013) presented how they used a web-based urban design reference tools to examine new opportunities for using precedents in urban design, and also found that that use of data rich 3-D digital models of precedents accelerated student learning, whilst Gunduz and Yetisir (2016) developed a design computer-based re-use application to be used in the steel work design for infrastructure projects.

However, it is suggested that, irrespective of how comprehensive they are, relatively few design rationale systems are actually used in the industry (Regli, et al., 2000). Therefore the authors of this paper have taken a step back and adopted a different approach: to produce a framework of design re-use criteria which healthcare designers can easily utilise to guide and facilitate the design re-use process itself. For healthcare design practice, such an approach is more effective compared to design rationale systems for a number of reasons. Firstly, design solution repositories are originally produced as a mean of capturing and storing organisational knowledge internally, so that they will be used effectively by others who then come into the organisation. It is unlikely that organisations will share their knowledge repositories with externally due to reasons associated with competitive advantage 
of knowledge capital, political repercussions or even security breaches if policy makers make their rationale available to the public. Secondly, it has been claimed that passive codified knowledge is less used during concept development or creation of new solutions because it is unable to instruct designers what actions to take in specific new design situations (Boling, 2010; Heylighen et al., 1999; Neuckermans and Fontein, 2002; Lawson, 2004). Thirdly, there are challenges associated with the development of such knowledge repositories. It takes considerable time, cost and effort to capture design solutions along with their design rationale (Sundaravadivelu et al., 2014) in part because, designers' intentions, rationale or design development information is rarely recorded in any form (Maher and Garza, 1996), or hidden in notes taken in hard copy notebooks with limited sharing (Baxter et al., 2008) and indeed inherently architecture is arguably rich, multi-layered, and hard to decode (Sönmez 2018). Finally, since healthcare designs are complicated and less repetitive, healthcare design solutions may need to undergo a significant level of alterations during the process of re-use (Wanigarathna et al., 2016). Healthcare designers need better skills of unpicking healthcare design problems (design requirements) associated with unique combination of patient needs, priorities, requirements and resources.

This paper proposes a framework that can be used by designers at project level in order to evaluate previous design solutions and capture necessary supplementary information to facilitate effective re-use. Instead of spoon-feeding or overloading designers with a selected number of design solutions, the framework proposed by this paper will help to effectively re-use design solutions gathered or identified through all other external sources, and not just design repositories. This approach would also facilitate double-loop (i.e. correcting the underlying causes behind the problematic action) learning environment as proposed by Love et al. (2016). 


\section{Research Methods}

\section{Research Design}

This research took a multiple case study approach as described by Yin (2009). The use of case studies enabled the collection of focused, in-depth, and rich data to allow the 'unpicking' of the project-unique circumstances associated healthcare built environment designs and type of detail that should be captured along with design solutions for effective reuse. Three case study projects were identified through industry partners, and were purposely selected based on a number of criteria. Recently completed (at the time of data collection ) projects were selected, to readily enable access to those involved during the design stage, and to ensure designers' ability to remember details related to designing the particular facility. Furthermore, as this research sought to evaluate successful design elements and design elements with weaknesses, in order to extract the type of detail that should be captured along with external design solutions, such elements and associated data necessarily had to be readily available within the Case Studies themselves. Ultimately this resulted in the selection of three case studies, within which the collection of data related both to the process of designing as well as the operational performance of the building could be undertaken by the researchers. Whilst this approach could potentially influence the validity and reliability of the case study selection process, the need for such detailed and relevant data, and for that data to be accessible to the researchers, was also a necessary criteria for consideration, and so in part drove the case study section from pragmatic perspectives. Details of the selected case study projects can be found in Table 1.

Table 1. Details of the Case Studies

\begin{tabular}{|l|l|l|l|}
\hline Project name & Case study A & Case study B & Case study C \\
\hline Type of the facility & A children's hospital & $\begin{array}{l}\text { Non-critical elderly care and } \\
\text { mental health hospital }\end{array}$ & Elderly care facility \\
\hline Location & Central London & Ebbw Vale & Bradford \\
\hline
\end{tabular}




\begin{tabular}{|l|l|l|l|}
\hline Type of construction & $\begin{array}{l}\text { A new modular building within } \\
\text { an existing hospital site }\end{array}$ & $\begin{array}{l}\text { A new building on a new site } \\
\text { an existing hospital site }\end{array}$ \\
\hline Purpose of the facility & $\begin{array}{l}\text { To replace some old facilities } \\
\text { and to increase the capacity }\end{array}$ & $\begin{array}{l}\text { To replace a number of existing } \\
\text { hospitals with three new } \\
\text { facilities to be operated under } \\
\text { a new care model }\end{array}$ & $\begin{array}{l}\text { To increase the capacity to } \\
\text { cope with winter pressure }\end{array}$ \\
\hline
\end{tabular}

Based on an initial set of discussions with design team leaders of the three Case Studies, 8 purposely selected exemplar design elements were initially selected for further exploration.

These 8 elements were selected to allow the maximum possible variety. Since, the research aimed for a generalisable framework, a careful effort was made to select design elements related to both the conceptual design phase and detail design phase; and elements related to both architectural design and engineering design. These elements represented both innovative or bespoke design problems as well as traditional or standard design problems which are frequently supported by design re-use. This approach was intended to ensure a relevant data set, but with minimal bias therein, and to ensure that the details and rationale that negated the re-use of designs (i.e. bespoke designs) were also captured.

The eight key 8 elements were Single bed room design (including en-suite and bed head services panel); Ward layout and clinical workstation design; Ventilation strategy and window design; Communal spaces design; Isolation room design; Floor wall and ceiling finishes; Design of doors; and Water service design. Within these 8 key elements, 78 sub design elements (Case Study A =27, Case Study B =25 and Case Study C =26) were investigated to collect data related to the design process and design rationale, including all aspects of re-use. These sub-design elements reflect the individual design component elements within each of the 8 aspects above. For example, design of doors had sub-elements such as door finish, designing a finger (not) trapping solution, and the design of the observation panel for single room doors. Therefore there were 78 units of analysis within the dataset as a whole. 


\section{Data collection method}

Most research exploring design knowledge re-use have mobilised 'think aloud' or similar methods to capture the designers' rationale during the actual design process. Such methods are used to help create design solution repositories to store design solutions along with supplementary information to facilitate subsequent effective re-use. However, as discussed in the previous sections, there are limitations associated with the use of such systems. The aim of this research is to propose a framework that can be easily used by designers at project level, to effectively evaluate and re-use external solutions contained in various sources to unique healthcare design problems. Such guidelines should be generic and suitable to evaluate any external design solution. This research adopted an approach that sought the explanations of design rationale provided by designers of 78 selected design elements, which formed the data for the study and used to develop the framework proposed in this research. In particular, data related to:

- the process of design and designers' rationale during the design of the78 elements,

- an evaluation of the 'success' of those design elements and

- the lessons learnt from successful and less successful solutions .

The data were collected over a period of 6 months. Data related to design rationale were gathered through interviews with the designers involved in the design of each element, and documentary analyses (such as presentations to the design team, various reports such as design alternative appraisals). The data related to the success of design elements and lessons learnt (the second and third aspects as noted above) were gathered through interviewing (on subjective opinion) design team leaders and the client representatives. As these were retrospective Case Studies, a clear limitation of the study was its reliance on memory recall of the interviewees and the accuracy and completeness of documentary records. Careful consideration and effort was made to gather data from multiple sources and potentially 
differing viewpoints, to mitigate the impact of this limitation and to improve the validity of data as a whole.

\section{Data analysis}

Interview data from all sources was transcribed and collated with other supplementary data to compile and create 'element stories' for each of the 78 design elements.

Extent of design re-use: Element stories were analysed based on the principles of deductive thematic analysis to determine the extent of design re-use using the following pre-determined coding framework.

a) Re-use of previously used design solutions gathered from various sources with minor or nor improvements, and

b) Designed bespoke solutions to solve design problems (no re-use).

c) Design solutions re-used with significant improvements,

d) Design solutions re-use that were initially considered and then rejected in favour of the design of bespoke solutions to solve design problems.

Rationale during the designs re-use: The element stories were then subjected to further analysis, again based on the principles of inductive thematic analysis, to develop codes and themes (Miles and Huberman, 1994; Hsieh and Shannon 2005) reflective of the aspects that needed to be considered for effective design re-use. These were revealed within the data as related to type of information from design considered (or not resulting failures) within their rationale (codes). These codes were then further examined to identify emerging patterns which denoted types of supplementary information that should be captured and considered during the design re-use (themes - 18nr). These themes were then grouped into key themes (5nr) which revealed mulita-criteria that should be considered during effective design re-use. 


\section{Results}

\section{Extent of design re-use}

Table 2 below shows the results of the deductive thematic analysis as focused on the extent of design re-use among three Case Studies and the 78 exemplar design elements (Case Study A =27, Case Study B =25 and Case Study C =26).

Table 2: Extent of design re-use

\begin{tabular}{|c|c|c|c|c|c|}
\hline & $\begin{array}{l}\text { Number of } \\
\text { design } \\
\text { elements } \\
\text { within Case } \\
\text { Study A } \\
\end{array}$ & $\begin{array}{l}\text { Number of } \\
\text { design } \\
\text { elements } \\
\text { within Case } \\
\text { Study B } \\
\end{array}$ & $\begin{array}{l}\text { Number of } \\
\text { design } \\
\text { elements } \\
\text { within Case } \\
\text { Study C } \\
\end{array}$ & $\begin{array}{c}\text { Total number } \\
\text { of design } \\
\text { elements } \\
\text { within all } 3 \\
\text { Case Studies }\end{array}$ & $\begin{array}{c}\text { Use of the } \\
\text { approach as a } \\
\%\end{array}$ \\
\hline $\begin{array}{l}\text { Design re-use with } \\
\text { minor or no } \\
\text { improvements }\end{array}$ & 9 & 14 & 16 & 39 & 50 \\
\hline $\begin{array}{c}\text { Design re-use with } \\
\text { significant } \\
\text { improvements }\end{array}$ & 4 & 6 & 2 & 12 & 15 \\
\hline $\begin{array}{l}\text { Design re-use was } \\
\text { considered and } \\
\text { rejected during the } \\
\text { rationale } \\
\end{array}$ & 3 & 4 & - & 7 & 9 \\
\hline $\begin{array}{c}\text { No design re-use } \\
\text { (Bespoke designing) }\end{array}$ & 11 & 1 & 8 & 20 & 26 \\
\hline Total & 27 & 25 & 26 & 78 & 100 \\
\hline
\end{tabular}

As shown in the table, there was a significant level of design re-use within all three Case Studies. The design teams gathered evidence of previous good designs via journals or research evidence databases, visiting existing hospitals with therapeutic features, and networking with professionals engage in healthcare design or research.

Case Studies revealed that some design solutions could be modified to improve performance or should be rejected if not suitable. Results revealed that in $15 \%$ of instances re-used design solutions were significantly modified to achieve bespoke solutions and, in a few more instances (9\%), initially considered re-used design solutions were rejected and alternative solutions were designed. Re-used design solutions should be modified if needed to improve the design to suit new design/ requirements identified during the design evaluation; 
to improve the design based on research evidence; to tailor the design solution to projectunique circumstances and extra design requirements; to cater for local capacity requirements and staffing resource levels. For example, the en-suite of the single-bed patient room in Case Study B was identified from Department of Health design guidance, and was then improved to increase the size and add facilities to support elderly patient requirements. Unsuitable or less suitable re-used design solutions should be rejected and better solutions sought. These rejections could be associated with: unsuitability of design solutions for project-unique circumstances discussed in the Figure 1; lack of economical and spatial efficiency; noncompatibility with design guidance or regulations. For example, Case Study A needed door frames to be designed to prevent children's fingers becoming trapped between the door frame and the door. A variety of solutions available in the industry to avoid such finger trapping were considered by Case Study A, but none of which were able to satisfy the performance requirement. The design team instead devised a new solution working by collaboratively with a selected manufacturer.

\section{Multi-faceted rationale during the designs re-use}

Results from the case-study analysis revealed that the re-use of external design solutions for healthcare buildings should be evaluated through a number of different lenses. Thematic analysis identified 4 key design re-use criteria (themes) and sub-criteria (sub -themes) related to the type of information that should be captured and considered for effective design re-use (see Figure 1). The 4 key criteria are: patients' type and requirements, care models of the hospital, local departmental needs, and facility operational aspects, and each contained a number of sub-criteria as noted below in Figure 1. The next section explores each key design re-use criteria in more detail. 


\section{Effective re-use of good designs of healthcare buildings}

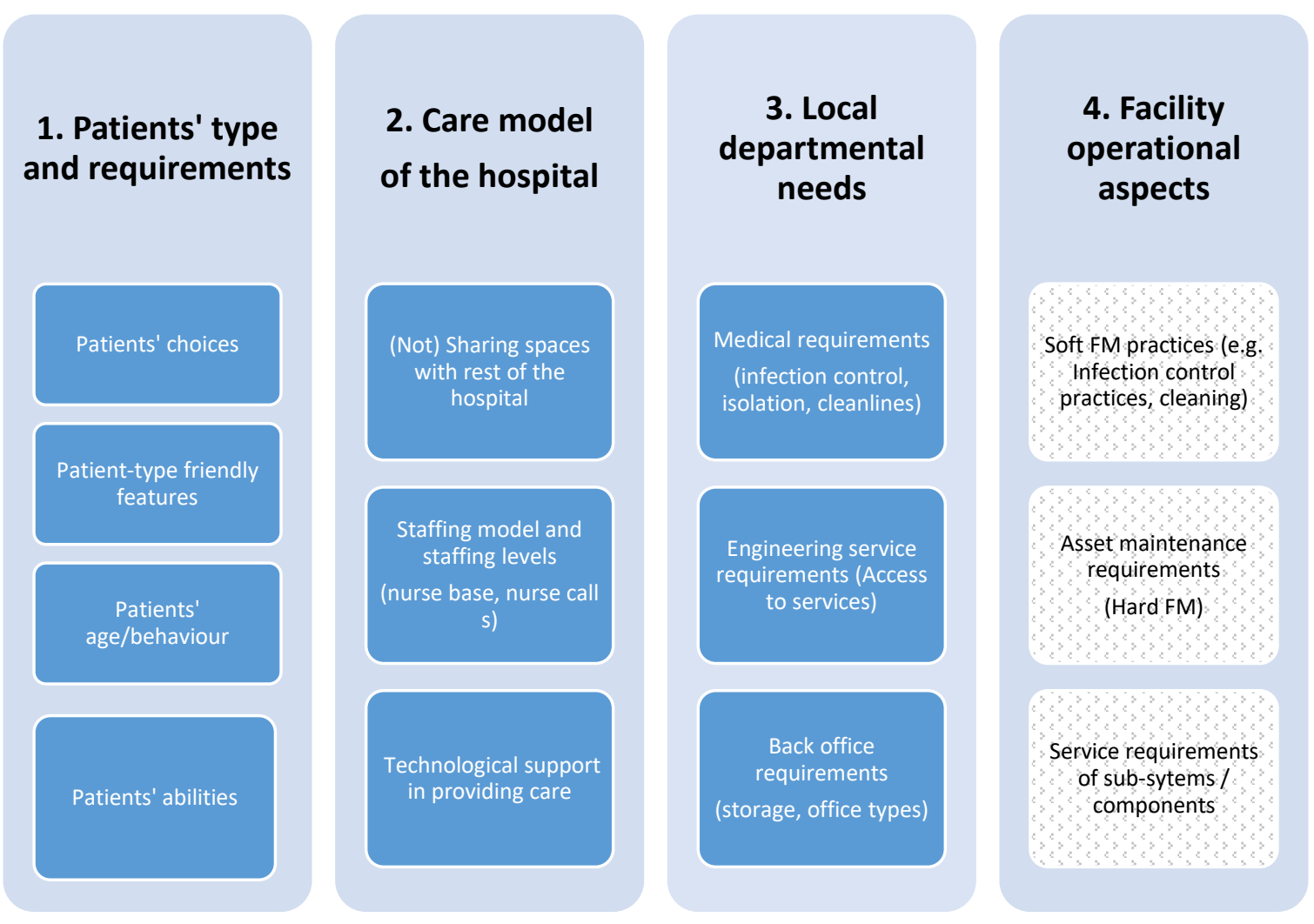

Figure 1: Thematic analysis of Effective design re-use criteria for healthcare buildings

Patients' type and requirements: All three Case Studies provided examples of the importance of reviewing the type of patients and their requirements as they were attached to the original designs. This facet of the rationale was essential in providing supporting evidence in order to successfully adapt them to suit the patients and their requirements of the prospective design. In particular due consideration should be given into patients' choices, patient friendly features, patients' age/behaviour and patients' abilities. Choices for different spaces of facilities is dependent on the type of patients. For example, elderly patients may prefer a bath over a shower room, and alternatively a shower room may work better for children. Therefore, Case Study A was designed with en-suite shower rooms for each patient rooms and Case Study B was designed with en-suite shower rooms and an additional 
bathroom per ward. The data revealed that designers had difficulties in predicting patients' behaviours and requirements in using facilities when similar solutions are re-used for a different patient group. For example, single-bed patient rooms in Case Study B were designed with windows that could be opened manually to support natural ventilation and energy savings. The design team were aware that manually opening windows could be difficult for elderly patients, yet this difficulty was deemed to outweigh the benefits related to natural ventilation and energy savings. However during the operation of the building, patients were contacting nurses through the nurse call system to get assistance in opening and closing the windows, which in turn caused the nurse call system to become both overloaded and congested, so nurses had difficulty in identifying urgent calls. A further example was found in Case study A, which instead adopted a mechanical opening strategy for windows at centrally set times, yet during the operation of the building it became apparent that the noise caused by mechanical opening of windows during the night was disrupting children's sleep. Care model of the hospital: Design features whether they be spaces or systems should be evaluated along with the care model of the hospital or department they are embedded in. Effectiveness of designs depend on shared spaces (such as isolation rooms, examination rooms) and level of sharing, staffing models and staffing levels (such as patient to staff ratio), and technological support in providing care (eg: aid of CCTV monitoring). For instance, Case Study A designed nurse stations for every four rooms cluster to observe children need speciality care, however, Case study B designed nurse stations for every eight rooms cluster to care for non-critical elderly care patients, considering staff for patient ratio.

Local departmental needs: Case Studies provide examples that in many cases healthcare built environment design problem will not accept a one-size fits all solution. All the departments have their own design requirements for built environments grounded on care provision requirements (such as level of isolation required), and the level of back office work 
related to each department. Further, some spaces have specific engineering service requirements (eg: access panels for engineering services). The quote below from a client representative for Case Study A explained what designers should have considered (and they did not) during the design of offices.

".........The office accommodation is used very well, by two specialities in particular, ICU probably don't use their offices as much as they could, so what they have done is they have created a couple of zones within the office........."

Facility operational aspects: Due consideration into facility operational aspects is key criteria for the success of re-using of design features. In particular, comparing and contrasting soft facilities management services (e.g. infection control practices, cleaning regime), hard asset maintenance requirements of the original design and prospective design is essential. Further, a due consideration should be given to the service requirements of associated subsystems or sub-components (e.g. life of batteries of sub-components). One example is the colour of wall finish of Case Study A. The design team had selected a particular white paint as a wall finish after visiting several newly built healthcare buildings. Later, during the operational phase, it was understood that a heavy cleaning regime is required to maintain cleanliness in the building and leave the wall finish in good condition.

Implementation of design solutions - culture of users: A further theme (nominally theme 5) that emerged from the data could be closely associated with the culture of the users themselves, and again incorporated several sub-themes: careful use, choice, use of spaces/design for intended purposes, leadership for staff and follow user instructions for systems. This theme suggests that careful use alone, as described in the previous sections does not make design re-use (and bespoke solutions) a success. Some external aspects related to the building users also impact the performance and success of the design. Careful use of 
the hospital and its features by staff, patient and visitors is a key external criteria of the performance of the building. For instance, if users operated doors cautiously, especially when passing through with trolleys and other equipment, damage to wood veneer finish could be minimised. All three Case Studies have provided a good level of control of the environment and comfort for the users (e.g. temperature control, control of the window opening). However, some building users look for the control of the environment and comfort levels related to every single feature of the building (e.g. local temperature control of water for nontouch taps). Furthermore, users if not familiar with some new spaces or features of the building (design) may not use them as intended and with optimum efficiency.

“....we are happy about [Case Study B] but they are still putting things in corridors like mobile hoists, and not using store rooms properly...."(a representative from the Client)

It was highlighted that the performance of the design during the operational phase could have been better if the building is considerately used by users and the design is effectively introduced to the users. Introducing the design to the hospital staff could be done in many ways, for example if existing staff participated during the design phase to evaluate the design to better understand the design rationale and become familiar with the design over time, or training could be more timely for new systems for the staff, for example how to operate 'Nurse call system'.

More importantly, champions and good leadership including positive attitude of senior management can lead staff to better accept and use the hospital design successfully. “.....it is interesting because you got the same design but two different staffing groups, I think system in the xxx was better they were prepared for it, but in $x x x x$ they did not like it from the start..., so it is not just down to design ....." (a representative from the Client - Case study B) 
when you show them to the people some people came up with reasons why

it will not work and others were saying that they are trying to change their job within

GOSH to go in to the new building to work, so it was both ways.....

\section{Development of the framework and discussions}

The results above highlight that design re-use should be entailed with a multi-faceted evaluation criteria as discussed above, and supported by evidence collected and evaluated via multi stakeholders. These include the hospital's care provision strategy, related information gathered from senior managers (patient types and requirements, care models of the hospital), evidence gathered on the functionality related aspects of the design features (Local departmental needs, culture of users) from medical professionals, and evidence on success of design features during the facility operational phase gathered from facilities management staff. In many instances designs were evaluated against multi-criteria, however it was noted that some of the design features and related decisions could have been improved had the design team adopted a multi-criteria approach and gathered evidence from a larger number of stakeholders. For instance, single bed patient rooms in children hospitals works well in terms of providing accommodation and time for the family to visit children (patient requirements senior managers). However, in shared-bed ward, mums/carers can learn how to care for speciality need patients observing and talking to similar patients and carers in the ward (local departmental needs - medical staff). There were instances in which solutions identified were evaluated for patient type, patient requirements, and local departmental needs but failed to evaluate design feature for facilities management related aspects. Reasons for this could be associated with reduced involvement of those parties during the design requirement formulation and design evaluation. Furthermore, in some instances, particularly for new hospitals, facilities management professionals are not appointed at the time of the designing. 
From all three Case Studies it was clear that all design (to a certain extent) was a redesign. As claimed by NHS Estates \& Facilities Policy Division (2013) due to the unique combination of patient needs, requirements and resources, and operational strategies, design solutions are re-used and adapted with the significant moderation to provide bespoke design solutions. In addition, multi-stakeholder involvement (designers, senior managers, medical staff, facilities management staff and patient) is essential for effective re-use of design solutions to identify the suitability of solutions, modification requirements and sub-sequent effects of design solutions during the operational phase. Finally, appropriate introduction of the design to the users is central to the success of implementation of design. The research identified how the culture of users can impact the success of the design and suggested how design should be optimally introduced to the users to ensure their support and engagement. Figure 2 below therefore brings together the results of this research into a framework for the effective re-use of good healthcare design, including the acknowledgement of the culture of the users as revealed by the research.

Overall, the multi-faceted design evaluation criteria that emerged through this research confirms the difficulty of developing comprehensive and effective design solution repositories for healthcare buildings. The original artefacts in their context provide rich web of evidence which could not be easily abstracted into effective design repositories. Therefore, design re-use can be considered as a project level activity where the design team should capture necessary supplementary information along with the designs they intend to re-use, and compare and contrast those evidence with the particulars of the new design problem in order to facilitate effective re-use. 


\section{Framework for the effective re-use of good healthcare designs}

\section{Evidence gathered and evaluated via multi-stakeholder involvement}

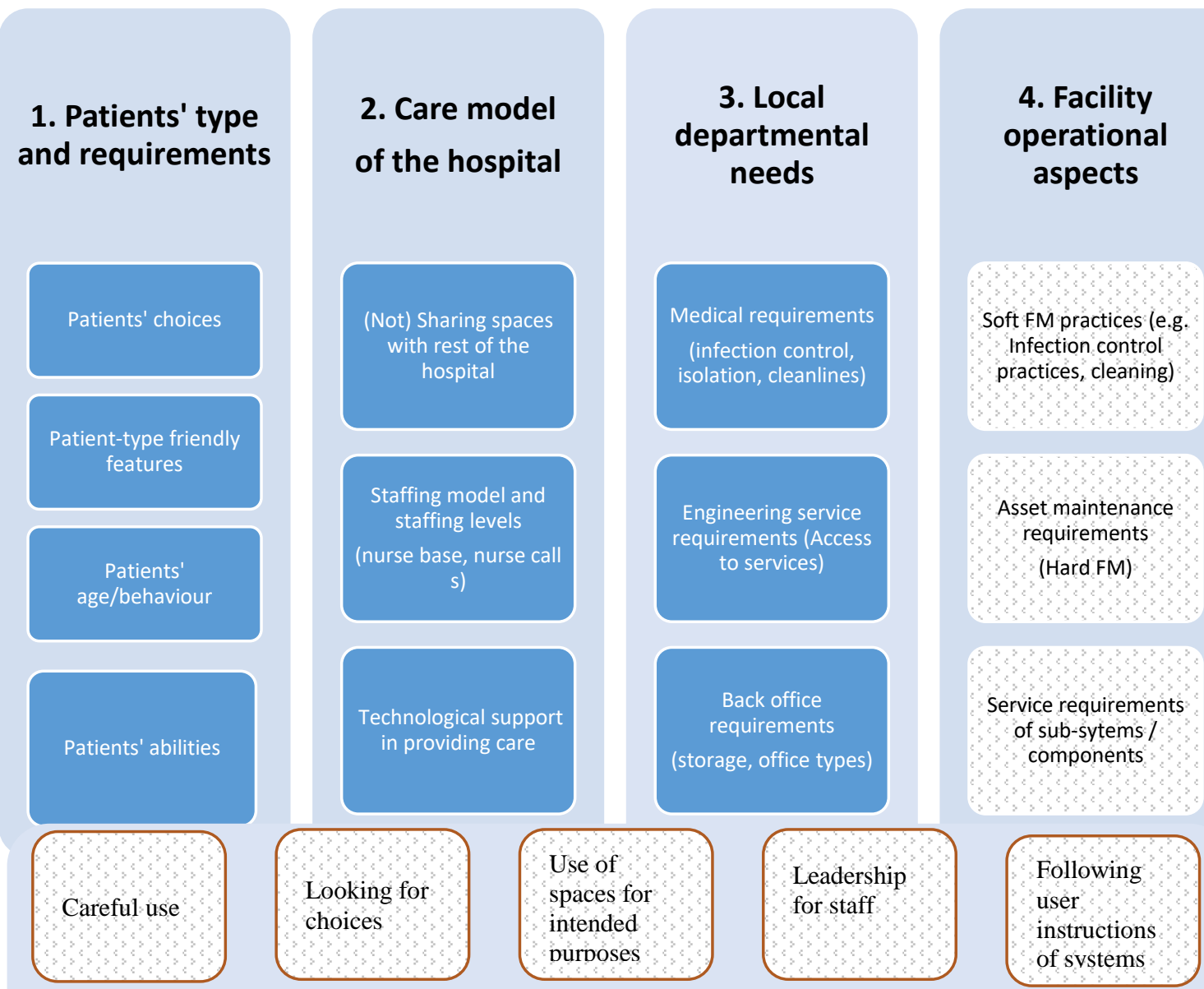

5. Culture of users

Figure 2: The Framework for the effective re-use of good healthcare designs

These results support the claims of Fruchter and Demian (2002) as related to the failures of passive knowledge due to the lack of access to contextual or informal knowledge. This study revealed that designers have gathered and evaluated a good level of contextual information during the re-use of healthcare designs, and in the majority this results in successful designs. The design team had been successful in considering many clinical related requirements and parameters (criteria 1-3 in Figure 2) but were less successful in the consideration of 
operational aspects (criteria 4 in Figure 2). Furthermore, they were less successful considering the culture of users and in introducing them to some of the key design elements. Reasons for this could be associated with reduced involvement of those parties (in particular facilities management staff and medical staff) during the design process.

[insert Figure 2 near here]

The results also confirmed Regli, et al. (2000)'s claim regarding the lack of effective and useful of details contained in the in-house design repositories, and highlighted that a successful design for one situation may not be successful unless it is carefully adapted. This is again due the multi-stakeholder involvement during the development and evaluation of healthcare design solutions. In essence it could be argued that both passive and active design knowledge should be careful re-used considering care related and operational aspects described in this research.

Overall, the results of this research are able to demonstrate that the approach taken by authors was effective in developing a project level framework that can be used by designers at project level, to effectively evaluate and re-use design solutions to solve unique healthcare design problems. The framework is particularly useful for the healthcare sector since design re-use entails a significant level of consideration related to care-provision parameters, which would not normally be stored in an architectural design repository.

\section{Implications for Practice}

The framework presented in this paper may help future research on design re-use and CBDSs to determine the type of information that should be captured and stored with good designs. Designers could use this frame work when re-using their own designs or design solutions identified externally, in order to evaluate the suitability of such designs and adapt them to suit the circumstances and constraints of new projects. It is also suggested that the resultant building and its systems should be appropriately introduced to the users in order to 
improve the acceptance and appropriate use of them on a day to day basis. Finally, for both research and practice, the findings suggested the importance of capturing design rationale as well as its performance during the in-use phase of the buildings.

\section{Conclusions}

There is a significant level of design re-use within healthcare built environment designs. Designers then reflect on these design solutions, along with evidence from the original design within the context of the new design problem, before they adopt, adopt or reject the solutions.

This research has demonstrated that designs re-use from both internal and external repositories should be compared and contrasted for number of different criteria related to patients' type and requirements, care models of the hospital, local departmental needs, and facility operational aspects attached to the original design and subsequent re-use situation. It was evident from the Case Study data that design teams pay limited consideration to the criteria related to facility operational aspects when compared to other three criteria and thus results in majority of design disappointments lie within that area.

This multi-faceted design evaluation process should be supported by the evidence collected and evaluated via a multi-stakeholder approach. These should include senior managers of hospitals to gather care provision strategy related evidence; medical staff to gather local departmental needs; facility managers, other operational staff and patients, to gather evidence related to operational aspects. Finally, the results also revealed that consideration of the criteria stated above alone does not make design re-use (and bespoke solutions) a success. Irrespective of the effort taken during the reasoning of the design solutions, properly introducing the design to the users plays a key role in success to tackle issues related culture of uses. 


\section{References}

Boling, E. (2010), "The Need for Design Cases: Disseminating Design Knowledge". International Journal of Designs for Learning, 1(1), pp 1-18.

Baxter, D., Gao, J. and Roy, R. (2008), Design process knowledge reuse challenges and issues. Computer-Aided Design and Applications, 5(6), pp. 942-952.

Demian, P., Ruikar, K., Sahu, T. and Morris, A. (2016), 3DIR: exploiting topological relationships in three-dimensional information retrieval from BIM environments. Yabuki, N. and Makanae, K. (Ed.), The 16th International Conference on Computing in Civil and Building Engineering. July 6-8, 2016, Osaka, Japan.

Domeshek, E., \& Kolodner, J. (1991), “Towards a case-based aid for conceptual design”. International Journal of Expert Systems, 4:2, 201-220.

Dorst, K. and Cross, N. (2001), "Creativity in the design process: co-evolution of problemsolution”. Design Studies, 22(5), pp. 425-437.

Edum-Fotwe, F., Gibb, A. and Benford-Miller, M., 2004. Reconciling construction innovation and standardisation on major projects. Engineering, Construction and Architectural Management, 11(5), pp. 366-372.

Emmitt, S. (2007), Design management for architects. London: Wiley-Blackwell.

Flemming, U. (1994), "Case-based design in the SEED system". Automation in Construction, 3(2-3), pp. 123-133.

Fruchter, R. and Demian, P. (2002), "Knowledge management for reuse". Paper presented at the CIB w78 Conference, 12 - 14 June 2002, Denmark, available at: https://www.researchgate.net/profile/Peter_Demian/publication/238703114_Knowledge_Man agement_for_Reuse/links/5444c9240cf2e6f0c0fbbc59.pdf (accessed 22 March 2018).

Grover, R., Emmitt, S. and Copping, A. (2017), The typological learning framework: the application of structured precedent design knowledge in the architectural design studio. International Journal of Technology and Design Education, 27 (1), pp. 1-20.

Gunduz, M. and Yetisir, T. (20160, A design reuse technology to increase productivity through automated corporate memory system. Neural Computing and Applications, 27 (1), pp. 1-9.

Hamilton, D.K. (2003), "The four levels of evidence-based practice”. Healthcare Design, 3(4), $18-26$. 
Hamilton, K., \& Watkins, D.H. (2009), Evidence based design for multiple building types. USA: John Wiley and Sons.

Heylighen, A., Bouwen, J.E. and Neuckermans, H. (1999), "Walking on a thin line between passive knowledge and active knowing of components and concepts in architectural design". Design Studies, 20(2), pp. 211-235.

Hsieh, H.F. and Shannon, S.E. (2005), "Three approaches to qualitative content analysis". Qualitative health research, 15(9), pp. 1277.

Hua, H. (2014), A case-based design with 3D mesh models of architecture. Computer-Aided Design, 57, pp. 54-60.

IDEAs, (2010), "IDEAs - Inspiring Design Excellence \& Achievements [Homepage of Department of Health], [Online]. Available: http://www.ideas.dh.gov.uk/ [08/10, 2010].

Kamara, J.M., Anumba, C.J., Carrillo, P.M. and Bouchlaghem, N.D. (2003) Conceptual framework for live capture and reuse of project knowledge [Homepage of Construction informatics digital library], [Online]. Available: http://itc.scix.net/data/works/att/w78-2003178.content.pdf [01/10, 2011].

Kululanga, K.G. and Kuotcha, S.W. (2008). Measuring organisational learning through project reviews. Engineering, Construction and Architectural Management, 15(6), pp. 580595.

Lawson, B. (2004), What designers know. UK: Architectural Press.

Love, P.E., Teo, P., Davidson, M., Cumming, S. and Morrison, J. (2016), Building absorptive capacity in an alliance: Process improvement through lessons learned. International Journal of Project Management, 34(7), pp. 1123-1137.

Maher, M.L. and Balachandran, B. (1994), "Multimedia approach to case-based structural design". Journal of Computing in Civil Engineering, 8(3), pp. 359-376.

Maher, M.L. and De Silva Garza, Gomez, A. (1996), "Developing case-based reasoning for structural design”. IEEE Expert, 11(3), pp. 42-52.

Maher, M.L. and Pu, P. (2014). Issues and applications of case-based reasoning to design. Psychology Press.

Main, J., Dillon, T.S. and Shiu, S.C. (2000), A tutorial on case-based reasoning. Pal, SK; Dillon, TS; and Yeung, DS

Miles, M.B. and Huberman, A.M. (1994), Qualitative Data Analysis: An Expanded Sourcebook. 2 edn. USA: Sage Publications, Inc.

Moore, K.D. \& Geboy, L. (2010), The question of evidence: current worldviews in environmental design research and practice. Architectural Research Quarterly, 14(02), 105114. 
Neuckermans, H. and Fontein, L. (2002), Nurture and nature of research in architecture, ARCC/EAAE Montreal conference on architectural research proceedings 2002, pp. 23-29.

Oxman, R. and Oxman, R. (1993), "Remembrance of things past: design precedents in libraries". Automation in Construction, 2(1), pp. 21-29.

Oxman, R.E., 1994. Precedents in design: a computational model for the organization of precedent knowledge. Design Studies, 15(2), pp. 141-157.

Oxman, R.E., 2010. Sharing media and knowledge in design pedagogy. Journal of Information Technology in Construction (ITcon), 15(22), pp. 291-305.

NHS estates \& facilities policy division. (2013), The NHS Premises Assurance Model (NHS $P A M)$. UK: Department of Health.

Regli, W.C., Hu, X., Atwood, M., Sun, W. (2000), A survey of design rationale systems: approaches, representation, capture and retrieval. Engineering with Computers, 16(3-4), pp.209-235.

Senaratne, S., Jin, X. and Balasuriya, K. (2017), Exploring the role of networks in disseminating construction project knowledge through case studies. Engineering, Construction and Architectural Management, 24(6), pp. 1281-1293.

Senbel, M., Girling, C., White, J.T., Kellett, R. and Chan, P.F. (2013), Precedents reconceived: Urban design learning catalysed through data rich 3-D digital models. Design Studies, 34(1), pp. 74-92.

Sönmez, N.O. (2018), A review of the use of examples for automating architectural design tasks. Computer-Aided Design, 96, pp. 13-30.

Stichler, J.F. (2007), "Research methods for Evidence based design". Health Environments Research and Design Journal, 1(1), pp. 11-20.

Stichler, J.F. (2010), Research or Evidence-Based design: Which process should we be using? Health Environments Research and Design Journal, 4(1), pp. 6-10.

Sundaravadivelu, S., Vaidyanathan, A. and Ramaswamy, S. (2014). Knowledge reuse of software architecture design decisions and rationale within the enterprise, Issues and Challenges in Intelligent Computing Techniques (ICICT), 2014 International Conference on 2014, IEEE, pp. 253-261.

Taha, D., Hosni, S., Sueyllam, H. (2004), A case based architectural design application for residential units. 1st ASCAAD International Conference, e-Design in Architecture Saudi Arabia. December 2004. 109-124.

Tan, H.C., Carrillo, P.M., Anumba, C.J., Bouchlaghem, N., Kamara, J.M. and Udeaja, C.E. (2007), Development of a methodology for live capture and reuse of project knowledge in construction. Journal of Management in Engineering, 23(1), pp. 18-26. 
Wanigarathna, N. (2014), Evidence-based design for healthcare buildings in England and Wales. Unpublished PhD thesis, Loughborough University, UK.

Wanigarathna, N., Sherratt, F., Price, A.D.F., Austin, S.A. (2016), Healthcare designers' use of prescriptive and performance-based approaches. , Architectural Engineering and Design Management Journal. 12 (6), pp. 427-441.

Wang, L., Shen, W., Xie, H., Neelamkavil, J. and Pardasani, A., 2002. Collaborative conceptual design — state of the art and future trends. Computer-Aided Design, 34(13), pp. 981-996.

Yin, R.K. (2009). Case study research: Design and methods. Thousand Oaks, CA: Sage Publications.

Yu, J., Cha, J. and Lu, Y. (2012), Design synthesis approach based on process decomposition to design reuse. Journal of Engineering Design, 23(7), pp. 526-543. 